\title{
THREE-DIMENSIONAL NUMERICAL ANALYSES OF LIQUID-METAL MAGNETOHYDRODYNAMIC FLOW IN MAGNETIC-FIELD OUTLET-REGION
}

\author{
Hiroshige Kumamaru* \\ *Dept. of Mechanical Engineering, Graduate School of Engineering, Himeji Institute of Technology \\ 2167 Shosha, Himeji-Shi, Hyogo-Ken 671-2201, Japan
}

\begin{abstract}
A three-dimensional numerical calculation has been performed on liquid-metal magnetohydrodynamic (MHD) flow through a rectangular channel in the outlet region of the magnetic field, including a region downstream the magnetic field section. The continuity equation, the momentum equation and the induction equation have been solved numerically by the finite difference method. Along the flow axis (i.e. the channel axis), the pressure decreases rapidly as a fully-developed MHD flow, drops more rapidly in the magnetic-field outlet-region, and finally decreases slowly as a normal non-MHD flow. The pressure recovery that was observed in the magnetic-field inlet-region does not appear in the magnetic-field outlet-region. The total pressure drop through the magnetic-field outlet-region is larger than that through the magnetic-field inlet-region. The flow velocity distribution also changes gradually from a flat profile of a fully-developed MHD flow to a parabolic profile of a non-HMD laminar flow.
\end{abstract}

Keywords: Fusion Reactor, MHD, Magnetohydrodynamics, Liquid Metal, Lorentz Force, Numerical Analysis

\section{INTRODUCTION}

In a design example of a fusion reactor power plant, a lithium-bearing blanket in which a great amount of heat is produced is cooled by liquid-metal lithium [1]. However, the liquid-metal lithium needs to pass through a strong magnetic field which is used to magnetically confine high-temperature reacting plasma in a fusion rector core. Hence, there exists a large magnetohydrodynamic (MHD) pressure drop arising from the interaction between the liquid-metal flow and the magnetic field. In particular, the MHD pressure drop may become considerably larger in the outlet and inlet regions of the magnetic field than in the fully-developed region inside the magnetic field for the reason mentioned below.

Figure 1 shows the coordinate system, the applied magnetic field and the induced electric currents in the outlet region of the magnetic field. The applied magnetic field is imposed in the $y$ direction, taking a constant value for $z=0 \sim z 1$, a linear decrease from $z=z 1 \sim z 2$, and a value of zero for $z=z 2 \sim z 0$, as shown in Fig. 1(a).

In the region of fully-developed MHD flow near $z=0$, the induced electric current which is produced by the vector product of flow velocity and applied magnetic field flows in the negative $x$ direction as shown in Figs. 1(b) and 1(cl). The induced current returns by passing through regions very near the walls (in an $x-y$ plane at the same $z$ ) where the flow velocity is nearly zero in the case of insulating walls. (The induced current can also pass through the walls in the case of conducting walls.) The induced current loop has a relatively large electrical resistance, since the current needs to flow in the thin regions near the walls. The Lorentz force which is caused by the vector product of induced current

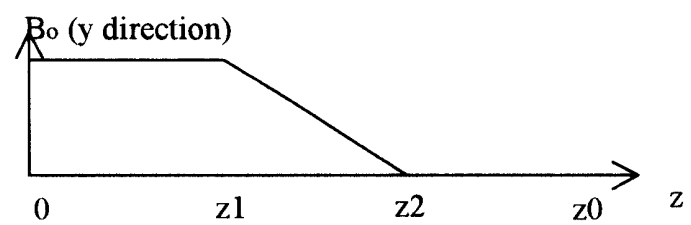

(a) Applied Magnetic Field

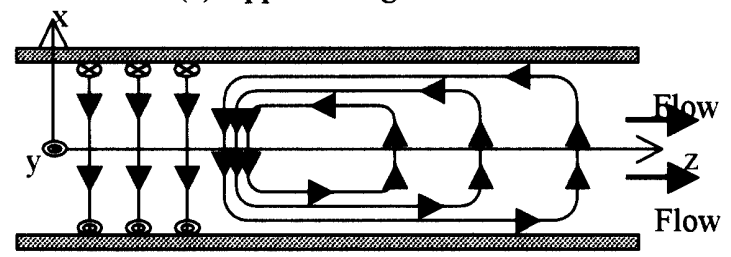

(b) Induced Currents in $x-z$ Plane

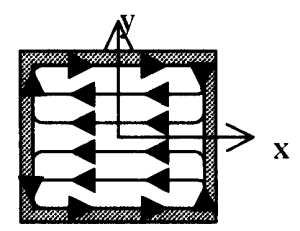

(cl) at $\mathrm{z}=0$

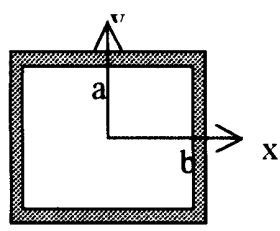

(c2) at $\mathrm{z}=\mathrm{z} 0$ (c) Induced Currents in $x-y$ Plane

Fig. 1 Coordinate System 
and applied magnetic field acts in the negative $z$ direction and produces a large pressure drop.

On the other hand, in the outlet region of magnetic field, the induced electric current flows in the negative $x$ direction, as was the case of fully-developed region, as shown in Fig. 1(b). However, the induced current can pass through the large region downstream the magnetic field section (in an $x-z$ plane with the same $y$ ) where no magnetic field or small magnetic field is applied. The electric resistance in this region is much smaller than the resistance in the thin region near the walls mentioned above. Hence, the induced current becomes larger in the outlet region than in the fully-developed region. The Lorentz force and thus the pressure drop also may become considerably larger in the outlet region than in the fully-developed region [2].

Figure 2 shows the applied magnetic field, the induced currents including the directions of Lorentz force and the pressure along $\mathrm{z}$-axis, in the inlet region and the outlet region of the magnetic field. The larger Lorentz force acts and thus the larger pressure drop occurs in the inlet and outlet regions than in the fully-developed MHD region. A smaller Lorentz force may act in the flow direction and thus a small pressure recovery may occur in the first section of the inlet region and in the last section of the outlet region, since the induced current flows in the positive $x$ direction [2]. The normal frictional pressure loss occurs in the regions of no magnetic field.

Several two-dimensional numerical calculations, in which infinite plates in the $x$-direction are assumed, have been performed so far on the MHD flow in the inlet region of the magnetic field (For example, refer to [3]). Furthermore, one approximate three-dimensional calculation, in which an approximate velocity profile is

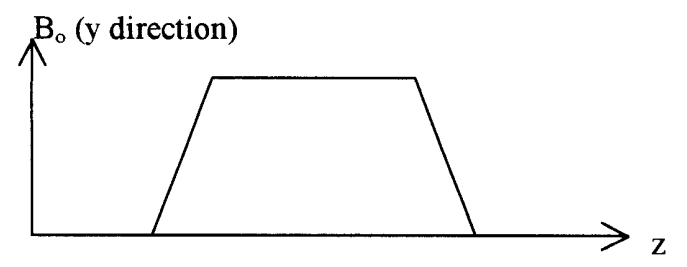

(a) Applied Magnetic Field

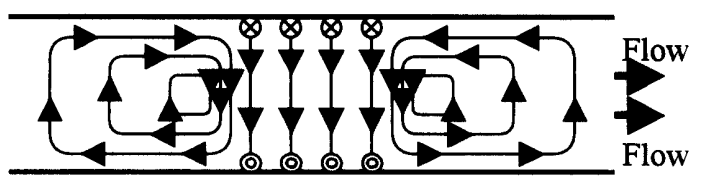

Lorentz $\Rightarrow \Leftarrow \Leftarrow \Leftrightarrow \Longleftrightarrow$

Force (b) Induced Current in $x-z$ Plane

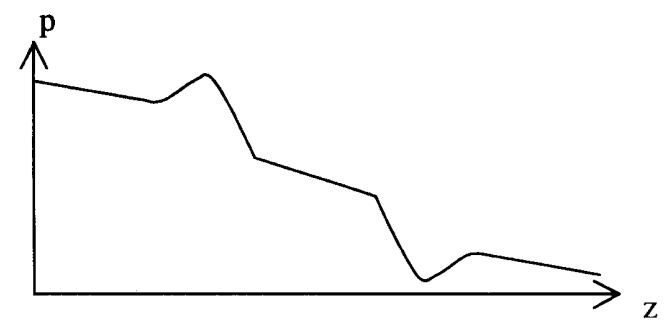

(c) Pressure along z-axis assumed near the walls perpendicular to the magnetic field, has been conducted on the MHD flow in the inlet region of the magnetic field [4]. However, the induced electric currents in the inlet region, mentioned above, flowing in $x$-, $y$ - and $z$ - directions cannot be calculated exactly by a twodimensional model or an approximate three- dimensional model.

For these reasons, the author has already performed three-dimensional numerical calculations on the MHD flow through a rectangular channel in the inlet region of the magnetic field, including the region of no magnetic field upstream the magnetic field region [5]. In this study, the author conducts a three-dimensional numerical calculation on the MHD flow in the outlet region of the magnetic field, including the region of no magnetic field downstream the magnetic field region, and compares calculated results with those for the inlet region of the magnetic field.

\section{NUMERICAL CALCULATIONS}

Numerical calculations are performed for an MHD flow in a rectangular channel, having a flow cross-section of $2 a \times 2 b$, shown in Fig. 1. A fully-developed MHD laminar flow enters the calculation domain at $z=0$, and a fully-developed non-MHD flow leaves the domain at $z=z 0$. The applied magnetic field is also imposed as shown in Fig. 1(a).

The basic equations which describe a liquid-metal MHD flow are the continuity equation, the momentum equation and the induction equation. The equations are expressed respectively by:

$$
\begin{aligned}
& \nabla \cdot \boldsymbol{v}=0 \\
& \rho\left[\frac{\partial v}{\partial t}+(\boldsymbol{v} \cdot \nabla) \boldsymbol{v}\right]=-\nabla p+\eta \nabla^{2} \boldsymbol{v}+\frac{1}{\mu}(\nabla \times \boldsymbol{B}) \times \boldsymbol{B}_{\boldsymbol{o}} \\
& \frac{\partial \boldsymbol{B}}{\partial t}=\nabla \times\left(\boldsymbol{v} \times \boldsymbol{B}_{\boldsymbol{o}}\right)+\frac{1}{\sigma \mu} \nabla^{2} \boldsymbol{B}
\end{aligned}
$$

The vector $\boldsymbol{B}$ is an induced magnetic field produced by the induced electric current, and is treated as an unknown variable together with the velocity $v$ and the pressure $p$. The third term in the right-hand side of Eq. (2) represents the Lorentz force. The induction equation, i.e. Eq. (3), is derived from Maxwell's equations and Ohm's law in electromagnetism.

The basic equations are expressed in nondimensional forms by introducing the following nondimensional variables and numbers:

$$
\begin{aligned}
& t^{*}=\frac{t}{a / \bar{w}}, x^{*}=\frac{x}{a}, y^{*}=\frac{y}{a}, z^{*}=\frac{z}{a}, \\
& u^{*}=\frac{u}{\bar{w}}, v^{*}=\frac{v}{\bar{w}}, w^{*}=\frac{w}{\bar{w}}, p^{*}=\frac{p}{\rho \bar{w}^{2}}, \\
& A^{*}=\frac{A}{\bar{w} \mu \sqrt{\sigma \eta}}, B^{*}=\frac{B}{\bar{w} \mu \sqrt{\sigma \eta}}, C^{*}=\frac{C}{\bar{w} \mu \sqrt{\sigma \eta}},
\end{aligned}
$$

Fig. 2 Inlet and Outlet Regions of Magnetic Field 


$$
\beta=\frac{b}{a}, R e=\frac{\rho \bar{w} a}{\eta}, H a=B_{o} a \sqrt{\frac{\sigma}{\eta}}, R_{m}=\frac{\bar{w} a}{v_{m}}
$$

The final nondimensional basic equations become:

$$
\begin{aligned}
& \nabla \cdot \boldsymbol{v}=0 \\
& \frac{\partial \boldsymbol{v}}{\partial t}+(\boldsymbol{v} \cdot \nabla) \boldsymbol{v}=-\nabla p+\frac{1}{\operatorname{Re}} \nabla^{2} \boldsymbol{v}+\frac{1}{\operatorname{Re}}(\nabla \times \boldsymbol{B}) \times \boldsymbol{H a} \\
& \frac{\partial \boldsymbol{B}}{\partial t}=\frac{1}{R m} \nabla \times(\boldsymbol{v} \times \boldsymbol{H a})+\frac{1}{R m} \nabla^{2} \boldsymbol{B}
\end{aligned}
$$

Star $\left(^{*}\right)$ is omitted to simplify the description in Eqs. (6) (8) and in the following description. Note that the Hartmann number $\boldsymbol{H a}=(0, H a(z), 0)$ is a vector in this study, though it is a known variable (a given parameter).

Considering the symmetry, the numerical calculations are carried out for the region of $0<x<\beta$ and $0<y<1$. (Note that the inner surfaces of the walls correspond to $x=\beta$ and $y=1$ in nondimensional coordinates.) As the boundary condition on the flow velocities, the inflow boundary condition is adopted at the flow inlet, i.e. at $z=0$, by fixing the fully-developed MHD flow velocity distribution which is obtained from calculations on fully-developed region [6]. The outflow boundary condition is given at the flow outlet, i.e. at $z=z 0$, by fixing the reference pressure.

As the boundary condition on the induced magnetic fields, $\partial \boldsymbol{B} / \partial Z=0$ and $\boldsymbol{B}=0$ are given at the flow inlet and the flow outlet, respectively. The former reflects the situation that the induced current does not change in the $z$-direction at the flow inlet, and the latter represents that no induced current exists at the flow outlet. Also at the walls, $\boldsymbol{B}=0$ is specified by assuming that the walls are electrically non-conducting. The boundary conditions on the induced magnetic fields at the symmetry plane of $y=0$ are not intuitively clear. Hence, by calculating for the whole region of $-\beta<x<\beta$ and $-1<y<1$, it has been confirmed that the conditions are given by:

$$
y=0: A(-y)=-A(y), B(-y)=B(y), C(-y)=-C(y),
$$

where $A, B$ and $C$ are the $x, y$ and $z$ components of $\boldsymbol{B}$, respectively. Note that $A$ and $C$ are odd functions of $y$ though $B$ is an even function of $y$. (At the plane of $x=0, A, B$ and $C$ are all even functions of $x$.)

The calculations are performed using a non-uniform expanding $10 \times 10 \times 20$ grid with gird elements closely spaced near the channel walls, particularly near the wall of $y=1$. Used is the staggered-grid formation in which the corresponding components of the velocity vector and induced magnetic field vector are arranged at the same positions. The discretization of the equations is carried out by the finite difference method, and the solution procedure follows the MAC method. The (first-order accurate) upwind differencing for the fluid convection terms in Eq. (7) is adopted.

\section{CALCULATION RESULTS}

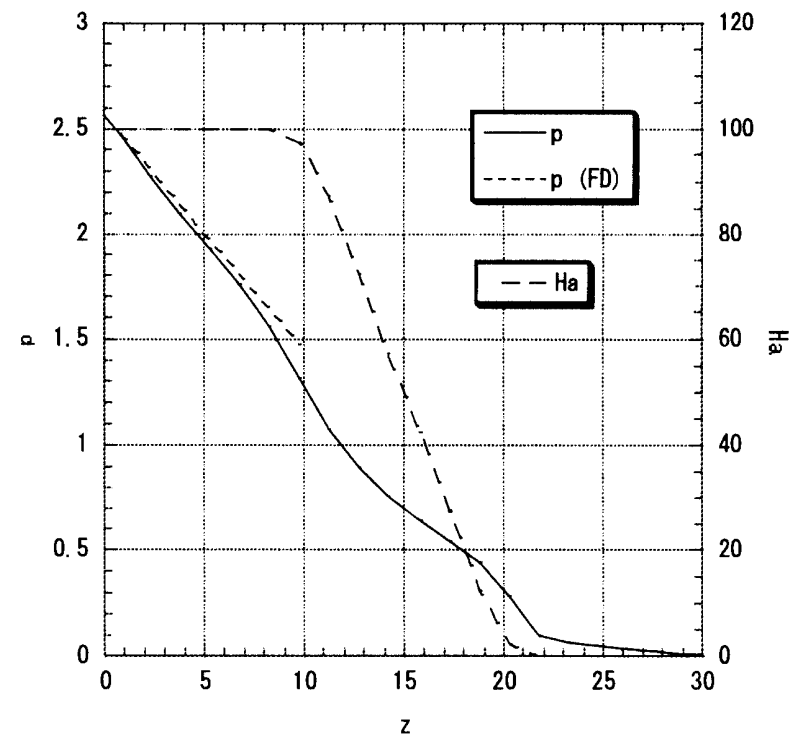

Fig. 3 Pressure along z-Axis

\subsection{Pressure Drop}

The calculation has been performed for a rectangular channel with insulating walls and an aspect ratio $(\beta)$ of 1.0 under the conditions of a Reynolds number $(R e)$ of 1000 , a Hartmann number $(\mathrm{Ha})$ of 100 (for the inlet fullydeveloped MHD region) and a magnetic Reynolds number $(R m)$ of 0.001 . The Hartmann number (relating to the applied magnetic field) is 100 from $z=0$ to 10 , decreases linearly from $z=10$ to 20 , and is zero from $z=20$ to 30 , i.e. $z 1=10, z 2=20$ and $z 0=30$ (See Fig. 1(a)). These values for the nondimensional numbers are selected to simulate those typical to experimental scales and conditions.

Figure 3 shows a calculated pressure along the flow axis, i.e. the $z$-axis, together with the change in Hartmann number. From $z=0$ to $z=\sim 8$, the pressure decreases rapidly representing the decrease as fully-developed MHD flow. The pressure drop in this region agrees nearly with a value calculated by the authors for the fully-developed region [6], which is shown in Fig. 3 as "FD". From $z=\sim 8$ to $z=\sim 12$, the pressure decreases more rapidly than in the region of $0<z<\sim 8$, since a larger Lorent $z$ force is produced in the negative $z$ direction as explained later. For $z=\sim 12$ to $\sim 22$, the pressure decreases rapidly but more slowly than in the region of $\sim 8<z<\sim 12$, since the Lorentz force acts in the positive $z$ direction at certain $y$ levels as explained later. However, no pressure recovery appears in this region. For $z>\sim 22$, the pressure decreases slowly following the decrease as normal non-MHD laminar flow.

There is no significant difference in the pressure within the $x-y$ cross section at a certain $z$.

\subsection{Velocity Distribution}

Figure 4 shows a calculated profile of the velocity $w$ from $z=0$ to $z=\sim 8$. There is no significant difference among the velocity distributions from $z=0$ to $z=\sim 8$. The velocity profile is a flat one having a peak value of 1.15 . The velocity distribution in this region agrees nearly with a 


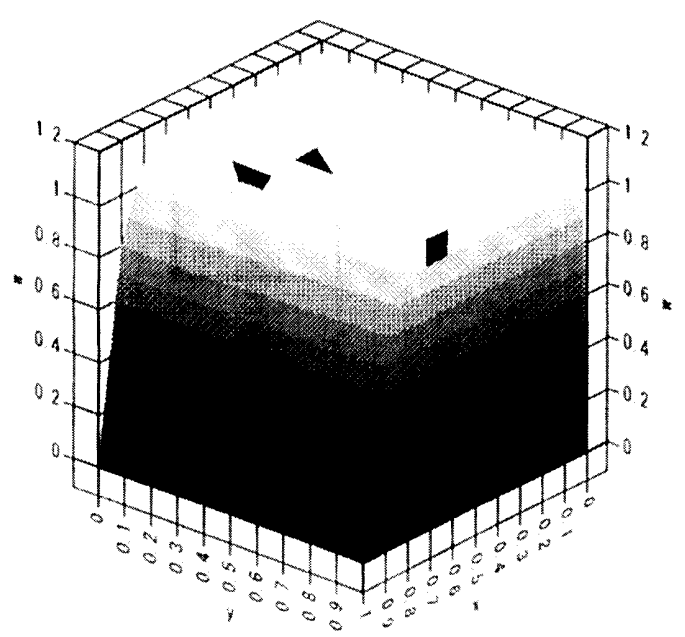

Fig. 4 Velocity $w$ Distribution at $z=0 \sim 8$

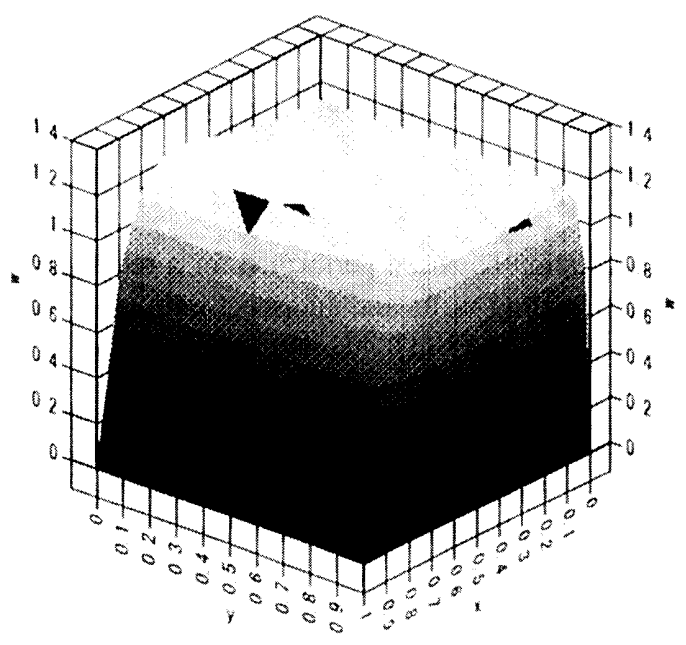

Fig. 5 Velocity w Distribution at $z=15$

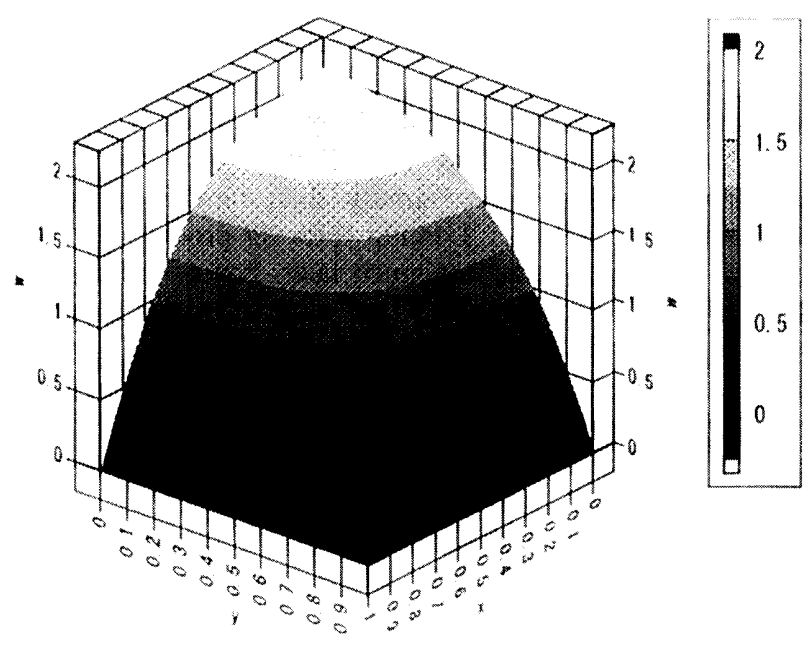

Fig. 6 Velocity $w$ Distribution at $\mathrm{z}=22 \sim 30$

profile calculated by the authors for the fully-developed MHD region [6].

Figure 5 presents a calculated velocity distribution at

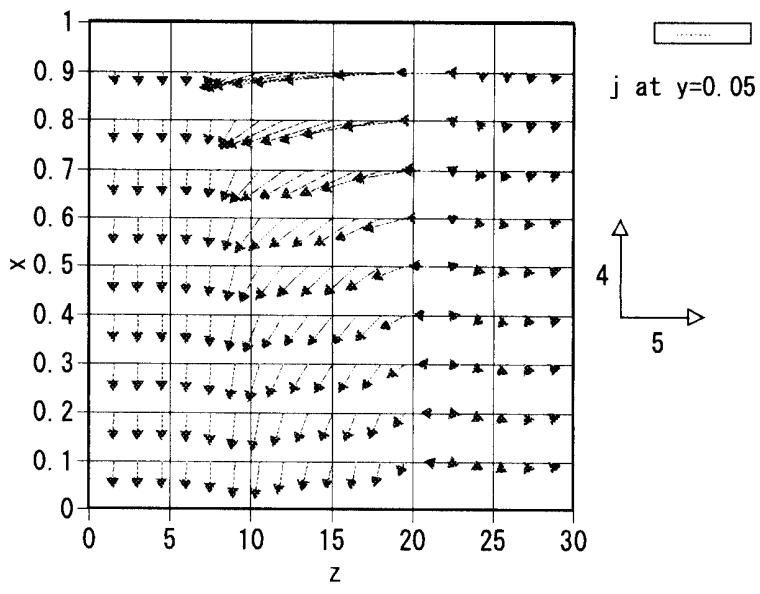

(a) At $y=0.05$

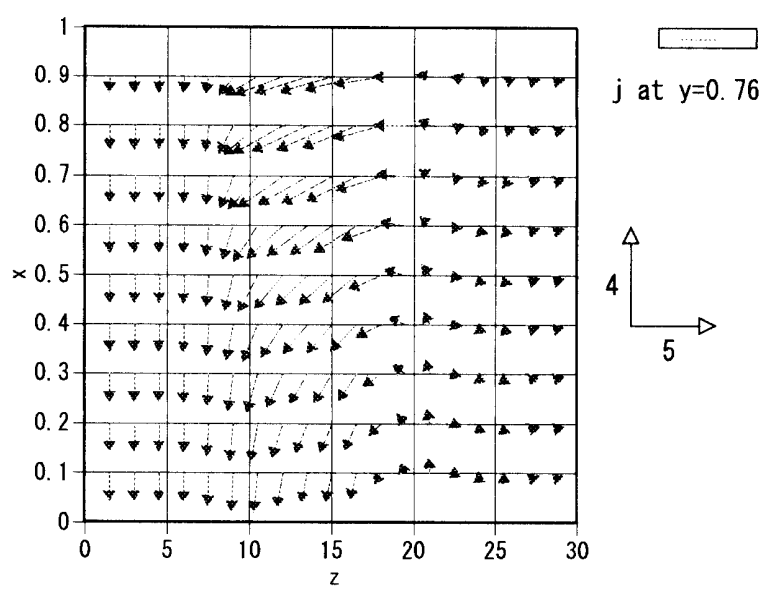

(b) At $y=0.76$

$z=15$. The distribution in Fig. 5 is still flat; however, the velocity near the wall of $x=1$ is larger than the velocity shown in Fig. 4. The velocity distribution changes gradually from a profile typical to an MHD flow to a profile typical to a non-MHD flow.

Figure 6 shows a calculate velocity profile from $z=\sim 22$ to $z=\sim 30$. No significant difference exists among the velocity profiles from $z=-22$ to $z=30$. The velocity distribution in Fig. 6 is a typical one of a laminar non-MHD flow with a peak value of $\sim 2$.

\subsection{Induced Current Distribution}

Figures 7(a), (b), (c) and (d) illustrate induced electric current distributions in the $x-z$ planes at $y=0.05$, $0.76,0.93$ and 0.99 , respectively. In the fully-developed MHD region of $0<z<\sim 8$, the induced current (flowing in the negative $x$ direction) is nearly the same, as shown in Figs. 7 (a) $\sim$ (c). This same induced current produces the same Lorentz force (acting in the negative $z$ direction) and results in the constant pressure drop along the $z$-axis. The induced current returns in an extremely thin region very near the wall of $y=1.0$, as shown in Fig. 7(d), i.e. the distribution at $y=0.99$.

In the outlet region from $z=\sim 8$ to $z=\sim 22$, the induced current forms a loop mainly in the $x-z$ plane, as shown in Figs. $7(a) \sim(c)$. The induced current is larger in the outlet region than in the fully-developed region. This is because 


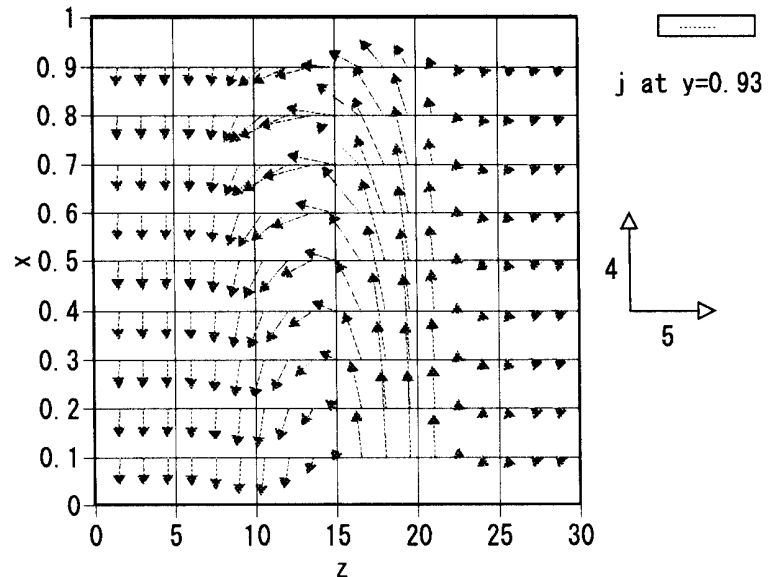

(c) At $y=0.93$

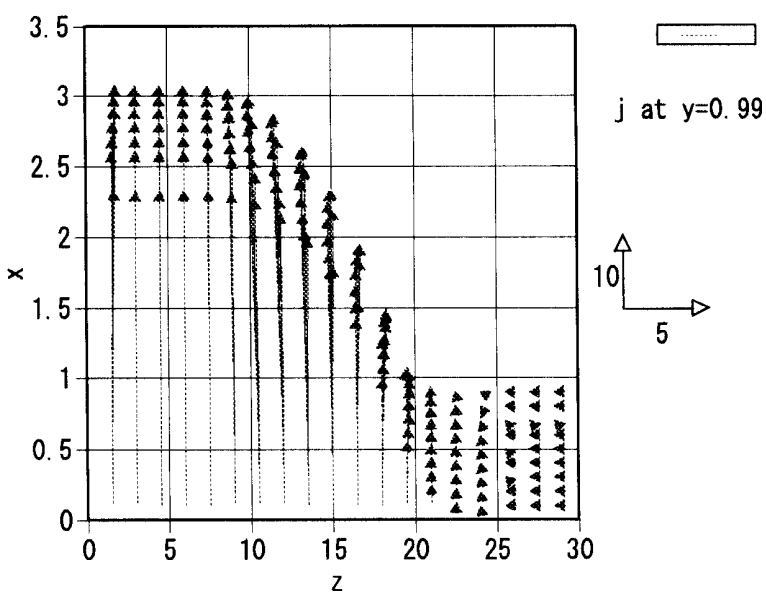

(d) At $y=0.99$

Fig. 7 Induced Current Distributions at $x-z$ Plane

the electric resistance of the induced current loop in the outlet region is much smaller than the resistance of the loop in the fully-developed region as mentioned in Introduction. In the region of $\sim 8<z<\sim 13$, the induced current flows in the negative $x$ direction at nearly all $\mathrm{y}$ levels. In this region, hence, a larger Lorentz force than in the fully-developed region acts in the negative $z$ direction, and a larger pressure drop is produced along the $z$-axis.

On the other hand, in the region of $\sim 13<z<\sim 22$, the induced current flows in the positive $x$ direction at higher $y$ levels. Thus, the total Lorent $\mathrm{z}$ force acting in the negative $\mathrm{z}$ direction becomes lower and thus the pressure drop along the $\mathrm{z}$ axis becomes smaller in this region than in the region of $\sim 8<z<\sim 13$. However, the pressure recovery does not happen in this region as mentioned in Sec. 3.1. There exist three-dimensional induced current flows with having more currents in the negative $x$ direction at lower $y$ levels and more currents in the positive $x$ direction at higher $y$ levels, as shown in Figs. 7(a) through (d).

In the region of no magnetic field, from $z=\sim 22$ to $z=30$, almost no induced current appears.

\subsection{Comparison of Outlet-Region with Inlet-Region}

Finally, the calculation results for the magnetic-field outlet region are compared with results for the

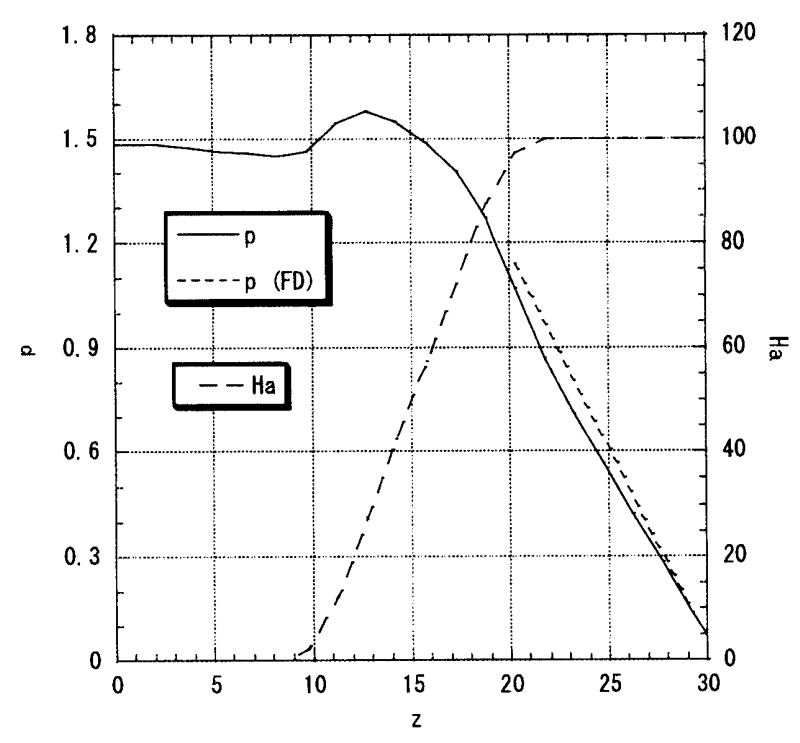

Fig. 8 Pressure along z-Axis for Inlet Region

magnetic-field inlet region. Figure 8 presents a calculated pressure along $z$-axis for the magnetic-field inlet-region, together with the Hartmann number along $\mathrm{z}$-axis [5]. The calculation parameters for Fig. 8 are the same as for Fig. 3, except for the Hartmann number change along $\mathrm{z}$-axis.

In Fig. 8, the pressure decreases slowly following the drop as normal non-MHD laminar flow from $z=0$ to $z=\sim 8$. The pressure recovery appears in the region from $z=\sim 8$ to $z=\sim 18$. This is because the induced current flows in the positive $\mathrm{x}$ direction and then the Lorentz force acts in the positive $z$ direction (See Fig. 2(b)). The pressure decreases more rapidly in the region from $z=\sim 18$ to $z=\sim 22$ than in the fully-developed MHD region of $z>\sim 22$. The reason is that the induced current in the negative $x$ direction and thus the Lorentz force in the negative $z$ direction are larger in the region from $z=\sim 18$ to $z=\sim 22$ than in the fully-developed MHD region of $z>\sim 22$ (See Fig. 2(b)). The pressure drop in this fully-developed region agrees nearly with a value calculated by the authors for the fully-developed region [6], which is shown in Fig. 8 as "FD".

The pressure recovery observed in the magnetic-field inlet-region (Fig. 8) does not appear in the magnetic-field outlet region (Fig. 3). The total pressure loss through the inlet section (Fig. 8) is smaller than that through the outlet region (Fig. 3).

In the present calculation on the liquid-metal MHD flow including large Lorentz force, the Poisson equation on pressures, i.e. a simultaneous linear equation on pressures, has been solved by the elimination method in order to get stable numerical solutions. (Stable numerical solutions have never been obtained by the iterative method in solving the Poisson equation.) Hence, the calculation has been performed with the coarse grid, i.e. $10 \times 10 \times 20$ (divided in $\mathrm{x}, \mathrm{y}$ and $\mathrm{z}$ directions, respectively) grid, due to the limitation of a computer capacity. A calculation performed with $10 \times 15 \times 30$ grid using a higher capacity computer shows almost the same results as those in the present calculation. 
The present calculation has also been performed for the calculation conditions which simulate typical experimental facility and conditions. It is desirable that a calculation will be performed for calculation conditions simulating a fusion reactor plant. That calculation may become possible by using a $20 \times 20 \times 30$ grid.

\section{CONCLUSIONS}

The three-dimensional numerical calculation has been performed on liquid-metal magnetohydrodynamic (MHD) flow through a rectangular channel in the outlet region of the magnetic field, including a region downstream the magnetic field section. The following conclusions have been obtained from the calculation results.

(1) Along the flow axis (i.e. the channel axis), the pressure decreases rapidly as a fully-developed MHD flow, drops more rapidly in the magnetic-field outlet-region, and finally decreases slowly as a normal non-MHD flow.

(2) The pressure recovery that was observed in the magnetic-field inlet-region does not appear in the magneticfield outlet region.

(3) The total pressure drop through the magnetic-field outlet-region is larger than that through the magnetic-field inlet-region.

\section{REFERENCES}

1. Asada, C. et al. Edited, Handbook of Nuclear Power, (1994), Ohm Company, Tokyo, pp. 791-793 (In Japanese).

2. Moreau, R., Magnetohydrodynamics, (1990), Kluwer Academic Publishers, Dordrecht-Boston-London, pp. 158-160.

3. Oki, Y. and Tanahashi, T., Entrance Flows of Electrically Conducting Fluids between Two Parallel Plates (Transients and Steady Flows), Trans. of JSME B, Vol. 58, (1992), pp. 14-21 (In Japanese).

4. Leboucher, L., Monotone Scheme and Boundary Conditions for Finite Volume Simulation of Magnetohydrodynamic Internal Flows at High Hartmann Number, J. of Computational Physics, Vol. 150, (1999), pp. 181-198.

5. Kumamaru, H., Three-Dimensional Numerical Analyses on Liquid-Metal Magnetohydrodynamic Flow in Magnetic-Field Inlet-Region, The 2002 ASME Pressure Vessels and Piping Conf., PVP-Vol. 448-1 (Computational Technologies for Fluid/Thermal/ Structural/Chemical Systems with Industrial Applications, Vol. 1), (2002), pp. 73-79, Vancouver, Canada.

6. Kumamaru, K. and Fujiwara, Y., Pressure Drops of Magnetohydrodynamic Flows in Rectangular Channel with Small Aspect Ratio and Circular Pipe for Very-Large Hartmann Numbers, Proc. of JSME/ASME/SFEN 7th Int. Conf. on Nuclear Engineering (ICONE-7), (1999), Paper No. 7041, Tokyo, Japan.
$A=x$ component of induced magnetic field

$a=$ half width of channel side parallel to applied magnetic field

$b=$ half width of channel side perpendicular to applied magnetic field

$\boldsymbol{B}=(A, B, C)$, induced magnetic field vector

$B=y$ component of induced magnetic field

$\boldsymbol{B}_{o}=\left(0, B_{o}, 0\right)$, applied magnetic field vector

$B_{o}=$ applied magnetic field

$C=z$ component of induced magnetic field

$\boldsymbol{H a}=(0, H a, 0)$, Hartmann number vector

$H a=$ Hartmann number

$p=$ pressure

$R e=$ Reynolds number

$R m=$ magnetic Reynolds number

$t=$ time

$u=x$ component of velocity

$\boldsymbol{v}=(u, v, w)$, velocity vector

$v=y$ component of velocity

$w=z$ component of velocity

$w=$ average of $w$

$\beta=b / a$, aspect ratio

$\eta=$ viscosity

$\mu=$ magnetic permeability

$v_{m}=1 / \sigma \mu$, magnetic kinematic viscosity

$\rho=$ density

$\sigma=$ electric conductivity

$*($ Star $)=$ nondimensional

\section{NOMENCLATURE}

